\section{Chefarzt-Kündigung Verschweigen von Strafverfahren kann fristlose Kündigung rechtfertigen}

Der Chefarzt hat eine herausragende Bedeutung für die Entwicklung und den Ruf einer Klinik. Daher enthalten Chefarztverträge oft eine Verpflichtung des Chefarztes, die Klinikleitung über Ermittlungen der Polizei und Staatsanwaltschaft zu informieren, damit sich die Klinikleitung gegenüber eventuell drohender schlechter Presse vorbereiten und verteidigen kann. Verletzt ein Chefarzt diese Pflicht, kann eine fristlose Kündigung gerechtfertigt sein.

\section{Der Fall}

Der Kläger ist habilitierter Facharzt für Gynäkologie und Geburtshilfe und bewarb sich im Jahr 2009 auf die von der Beklagten (einer Gesellschaft zur Gestellung medizinischer Mitarbeiter) ausgeschriebene Stelle eines Chefarztes zur Leitung der Abteilung Gynäkologie und Geburtshilfe in einer von der Gesellschaft betriebenen Kreisklinik. Die Parteien unterzeichneten am 22. Oktober 2009 den Dienstvertrag und das Arbeitsverhältnis begann am 01. November 2009.

Der Vertrag sieht unter anderem folgende Regelung vor: „Vorkommnisse von erheblicher oder grundsätzlicher Bedeutung, insbesondere auch Untersuchungen der Polizei oder der Staatsanwaltschaft, potenzielle Haftungsfälle, auftretende Schwierigkeiten oder Missstände in einer Abteilung hat der Arzt unverzüglich dem Dienstvorgesetzten - in ärztlichen Angelegenheiten über den leitenden Arzt der Kreiskliniken, im Übrigen auch über die Klinikleitung - mitzuteilen. Eine Korrespondenz mit Behörden, Versicherungen, Krankenkassen, MDK, Anspruchstellern, Presse und Rundfunk etc. findet ausschließlich über die Klinikleitung statt.“ Zudem unterzeichnete der Kläger folgende Erklärung zu Strafen und Disziplinarmaßnahmen: „Ich erkläre, dass ich über die vorstehenden Angaben hinaus nicht gerichtlich bestraft oder disziplinarisch belangt worden bin. Außerdem erkläre ich, dass gegen mich kein (weiteres) Strafverfahren, Ermittlungsverfahren der Staatsanwaltschaft oder Disziplinarverfahren anhängig ist.

Ich verpflichte mich, von jedem gegen mich eingeleiteten Straf- oder Ermittlungsverfahren und jeder gerichtlichen Verurteilung Mitteilung zu machen.“

Der Kläger war vor seiner Einstellung im
Jahre 2002 im Klinikum I GmbH beschäftigt. Eine Strafanzeige vom 25. November 2002 führte zur Aufnahme eines Ermittlungsverfahrens wegen fahrlässiger Tötung eines Neugeborenen gegen ihn. Hintergrund war der Vorwurf, er habe bei einem Geburtsvorgang am 17. November 2002 die Sectio (Kaiserschnitt) zu spät eingeleitet. Aufgrund des parallel laufenden zivilrechtlichen Verfahrens auf Zahlung von Schmerzensgeld setzte das Amtsgericht das strafrechtliche Verfahren zunächst aus. Nach der zivilrechtlichen Verurteilung des Klägers zur Zahlung von Schmerzensgeld an die Eltern des Neugeborenen nahm das Amtsgericht Anfang 2010 das Strafverfahren wieder auf und beschloss am 26. März 2010 die Eröffnung der Hauptverhandlung. Der Kläger erfuhr hiervon spätestens mit der Ladung im Mai 2010. Er wurde am 20. August 2010 wegen fahrlässiger Tötung zu einer Geldstrafe von 13500 Euro verurteilt.

Der Kläger informierte die Beklagte, also seinen neuen Arbeitgeber, weder über das zivilrechtliche noch über das strafrechtliche Verfahren. Die Beklagte wurde am Tag des Urteils durch Nachfragen der „Neuen I Zeitung“, die über den Prozess u.a. im Internet berichtet hatte, auf das Verfahren aufmerksam. Daraufhin suspendierte der Landrat noch am selben Tag den Kläger vom Dienst.

Mit Schreiben vom 23. August 2010 informierte der Kläger die Beklagte über die Einzelheiten des Zivil- und Strafverfahrens.

Nach Anhörung des Betriebsrates und deren Zustimmung zu der beabsichtigten Kündigung kündigte die Beklagte mit Schreiben vom 02. September 2010 das Arbeitsverhältnis mit dem Kläger außerordentlich, hilfsweise ordentlich zum nächstmöglichen Termin.

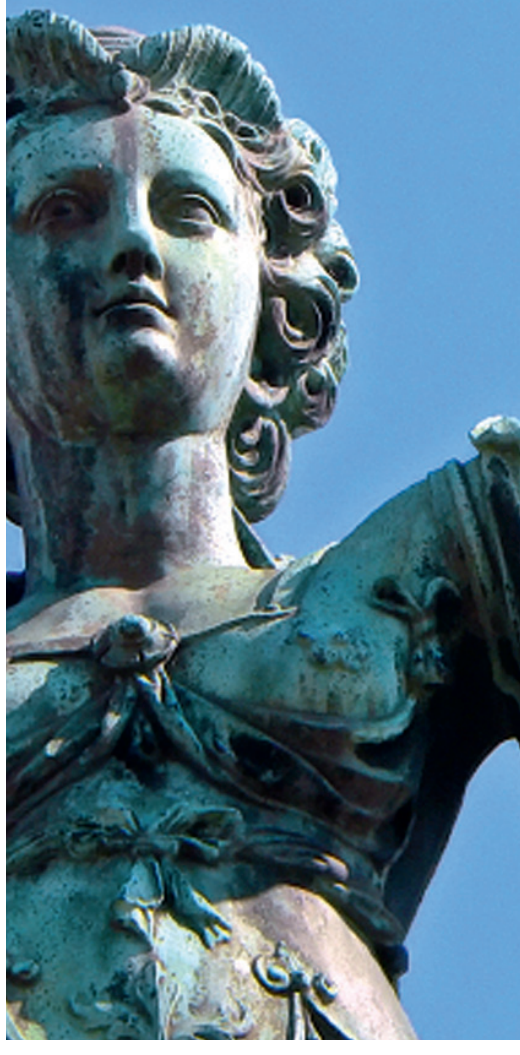

\section{Die Entscheidung}

Das Landesarbeitsgericht Hessen hob das erstinstanzliche Urteil des Arbeitsgerichts Darmstadt auf. Dieses hatte die Auffassung vertreten, dass das Arbeitsverhältnis nicht wirksam beendet wurde, weil weder ein wichtiger Grund für die außerordentliche Kündigung noch ein verhaltensbedingter Grund i.S.d. §1 KSchG vorliege. Dem folgte das Landesarbeitsgericht nicht. Es entschied, dass das Arbeitsverhältnis durch die außerordentliche Kündigung vom 02. September 2010 wirksam beendet wurde, da der Beklagten die Fortsetzung des Arbeitsverhältnisses aus wichtigem Grund nicht mehr zumutbar war.

Die Prüfung der Rechtmäßigkeit einer außerordentlichen Kündigung ist nach ständiger Rechtsprechung des Bundesarbeitsgerichts in 2 aufeinander folgenden Schritten durchzuführen.

\section{Ein wichtiger Grund liegt vor}

Es muss zunächst ein wichtiger Grund vorliegen, der abstrakt für sich betrachtet eine Kündigung rechtfertigt. 


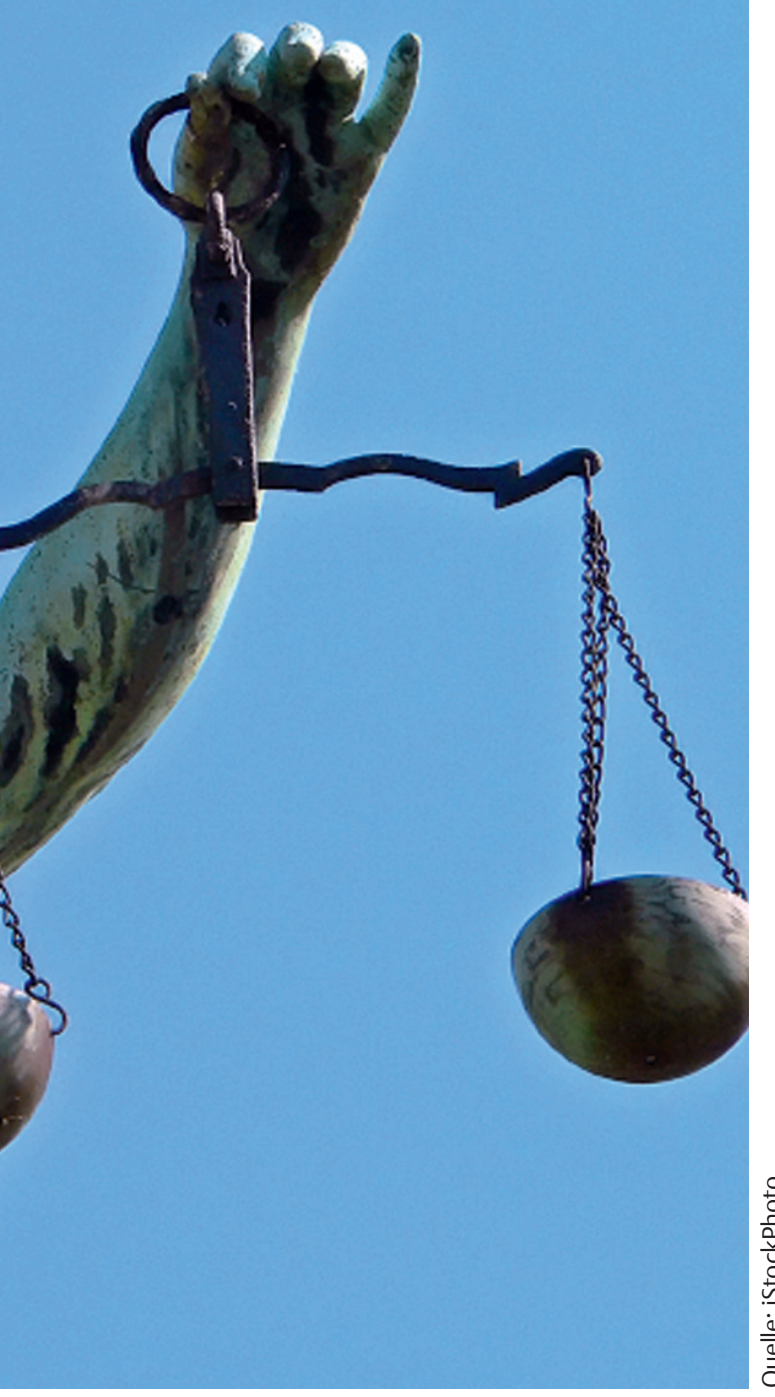

In dem Verhalten des Klägers, der es trotz ausdrücklicher und eindeutiger Verpflichtung unterließ, die Beklagte über das gegen ihn anhängige Strafverfahren wegen fahrlässiger Tötung in Kenntnis zu setzen, sieht das Landesarbeitsgericht einen wichtigen Grund an sich. Der Kläger habe sich in der von ihm unterzeichneten Erklärung verpflichtet, von jedem gegen ihn eingeleiteten Straf- oder Ermittlungsverfahren und jeder gerichtlichen Verurteilung Mitteilung zu machen. Im Mai 2010 wurde der Beklagte zur Hauptverhandlung des gegen ihn anhängigen Verfahrens geladen und hätte daher nach Ansicht des Landesarbeitsgerichts zwingend spätestens zu diesem Zeitpunkt seiner Verpflichtung aus der Erklärung nachkommen und die Klinikleitung informieren müssen. Das Gericht macht in ihren Entscheidungsgründen deutlich, dass dem Kläger unmissverständlich hätte deutlich gewesen sein müssen, welch hohen Stellenwert die Beklagte dem guten Leumund ihrer Beschäftigten, vor allem solchen in einer leitenden Position, bei- misst. Dies ergebe sich gerade aus der Tatsache, dass die Parteien nicht nur in $\S 6$ Nr. 8 des Arbeitsvertrages eine Meldepflicht hinsichtlich aller Vorkommnisse von erheblicher oder grundsätzlicher Bedeutung, zu denen ausdrücklich auch Untersuchungen der Polizei oder der Staatsanwaltschaft gerechnet wurden, vereinbarten, sondern die Beklagte daneben auch noch die Unterzeichnung der Erklärung vom Kläger verlangte. Darüber hinaus sah das Gericht in dem Umstand, dass die anhängige Strafsache einen Vorfall aus einem anderen Dienstverhältnis betraf, keinen Grund, der die Pflichtverletzung des Klägers schmälern würde. Die angeklagte Straftat stünde in einem so engen Bezug zur Tätigkeit des Klägers für die Beklagte, dass die Beklagte ein berechtigtes erhebliches Interesse an der Kenntnis der Umstände des Strafverfahrens habe. Zumal sei der Kläger wegen einer Tat angeklagt, die er bei Ausübung der Arbeit eines ärztlichen Geburtshelfers im Krankenhaus begangen hatte. Dies sei dieselbe Arbeit, die er auch nach dem Arbeitsvertrag mit der Beklagten dieser schulde. Auch der Zeitablauf ändere an der rechtlichen Beurteilung nichts, da dem Kläger im Jahr 2010, dem Jahr der Hauptverhandlung, bewusst sein hätte müssen, dass die Öffentlichkeit und gerade auch potenzielle Patientinnen vom aktuellen Strafverfahren erfahren und dadurch die von ihm geleitete gynäkologische Abteilung und letztendlich auch die gesamte Klinik negativ in Verbindung gebracht werden würde.

\section{Die Fortsetzung des Arbeitsver- hältnisses ist unzumutbar}

In einem zweiten Schritt ist im Rahmen einer umfassenden Interessensabwägung zu entscheiden, ob unter Würdigung aller Umstände des Einzelfalls dem Kündigenden die Fortsetzung des Arbeitsverhältnisses bis zum Ende der ordentlichen Kündigungsfrist unzumutbar war.

Das Landesarbeitsgericht kommt zu dem Ergebnis, dass dieses Kriterium vorliegt, da es für die Beklagte unzumutbar sei, die Frist der ordentlichen Kündigung abzuwarten. Die Pflichtverletzung gegenüber der Beklagten sei zu dem Zeitpunkt der Kündigung höchst aktuell und von weitreichender Bedeutung gewesen. Vor allem habe die Beklagte „auf Grund der Tatsache, dass der Kläger als Chefarzt eine durchaus herausragende Bedeutung für die Entwicklung und den Ruf der Klinik hat, ein alles überwiegendes Interesse daran, sich von einem Mitarbeiter in dieser Position sofort zu trennen, der nicht nur wegen eines in einer ähnlichen Funktion begangenen Tötungsdelikts verurteilt wurde, sondern es trotz ausdrücklich übernommener Verpflichtung unterließ, ihr von dem Verfahren Mitteilung zu machen.“ Durch das Unterlassen dieser Mitteilung sei das Vertrauensverhältnis derart zerstört, dass die Fortsetzung des Arbeitsverhältnisses unzumutbar sei, da eine Wiederherstellung des Vertrauensverhältnisses, welches für ein intaktes Arbeitsverhältnis unabdingbar sei, nicht wieder hergestellt werden könne.

Das Gericht erachtete eine vorherige Abmahnung für nicht notwendig. Vielmehr hätte dem Kläger aufgrund der ausdrücklichen Erklärung die überragende Bedeutung und der große Wert, den die Beklagte auf eine entsprechende Mitteilung legte, bewusst sein müssen.

\section{Fazit}

Der Chefarzt leitet die Fachabteilung in der Klinik. In dieser Position kommt ihm eine herausragende Bedeutung $\mathrm{zu}$, die auch darin besteht, dass er das Krankenhaus mit seinem Ruf repräsentiert. Der Ruf eines Chefarztes ist stets eng verknüpft mit dem Ruf des Krankenhauses. Daher sollte ein Bewerber bzw. Arbeitnehmer, gerade in führenden Positionen, in Bezug auf eventuell anhängige und einschlägige Verfahren offen umgehen und diese der Klinikleitung regelmäßig mitteilen. Ansonsten kann die Verletzung einer vermeintlich zweitrangigen Nebenpflicht zu einer wirksamen außerordentlichen Kündigung führen, insbesondere wenn es vertraglich geregelt ist.

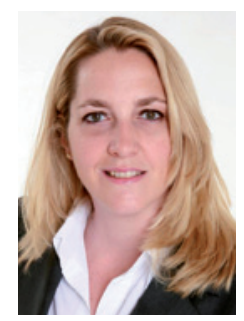

Korrespondenz Dr. iur. Isabel Häser Rechtsanwältin ECOVIS Lüdemann Wildfeuer \& Partner Sonnenstr. 9 80331 München www.ecovis.com 